\title{
Robust Nonlinear Control with Compensation Operator for a Peltier System
}

\author{
Sheng-Jun Wen, ${ }^{1}$ Dong-Yun Wang, ${ }^{1}$ Lei Zhang, ${ }^{1}$ and Ming-Cong Deng ${ }^{2}$ \\ ${ }^{1}$ School of Electrical and Information Engineering, Zhongyuan University of Technology, Zhengzhou 450007, China \\ ${ }^{2}$ Department of Electrical and Electronic Engineering, Tokyo University of Agriculture and Technology, Tokyo 183-8538, Japan \\ Correspondence should be addressed to Sheng-Jun Wen; wensj7062@gmail.com
}

Received 19 February 2014; Revised 4 June 2014; Accepted 26 June 2014; Published 9 July 2014

Academic Editor: Asier Ibeas

Copyright (c) 2014 Sheng-Jun Wen et al. This is an open access article distributed under the Creative Commons Attribution License, which permits unrestricted use, distribution, and reproduction in any medium, provided the original work is properly cited.

\begin{abstract}
Robust nonlinear control with compensation operator is presented for a Peltier actuated system, where the compensation operator is designed by using a predictive model on heat radiation. For the Peltier system, the heat radiation is related to the fourth power of temperature. So, the heat radiation is affected evidently by the temperature when it is high and temperature difference between the system and environment is large. A new nonlinear model with the heat radiation is set up for the system according to some thermal conduction laws. To ensure robust stability of the nonlinear system, operator based robust right coprime factorization design is considered. Also, a compensation operator based on a predictive model is proposed to cancel effect of the heat radiation, where the predictive model is set up by using radial basis kernel function based SVM (support vector machine) method. Finally, simulation results are given to show the effectiveness of the proposed scheme.
\end{abstract}

\section{Introduction}

With the rapid development of semiconductor materials, semiconductor refrigeration has been paid more and more attention in refrigeration research and industrial fields. And because of some advantages, such as no refrigerant, no pollution, no vibration, and no noise, semiconductor refrigerator is gradually going from lab to practical engineering, such as national defense industry, agriculture, and medical treatment [1]. Semiconductor refrigeration is also called electronic refrigeration or thermoelectric refrigeration, which uses special semiconductor material with composition of $N$-type semiconductor and $P$-type semiconductor. That is, it is a new type of refrigeration method through the input direct current flow, and it has been one of the three typical refrigeration ways in the world. In the semiconductor refrigeration, Peltier device is usually used as one of the refrigeration components and aluminum plate is considered as the thermal sink. Due to the Peltier effect, heat is absorbed at one end and pumped out through the other end when an external voltage is applied to a $N$-type/ $P$-type thermal couple. Therefore, a Peltier actuated aluminum plate thermal system is often utilized to analyze the refrigeration performance of the Peltier device.
In the Peltier actuated system, heat exchange mainly includes three forms: heat convection, heat conduction, and heat radiation. By modeling using some thermal exchange laws, it shows that the Peltier actuated system is a typical nonlinear affine system, where input and output are current and temperature, respectively. Not only the system temperature depends on input current and its square, but also there exists the fourth power of the temperature in the model. For the nonlinear system ignoring heat radiation, operator theory based nonlinear control method is confirmed to be effective to ensure robust stability of the controlled system [29]. Operator theory is a control theory based on an idea that a signal in the input space is mapped to the output space, which can be applied in linear system and nonlinear system [3]. So far, a lot of researchers have paid attention to the nonlinear control in regard to the theory, and some interesting results have been concluded [2-9]. An outstanding example is that robust nonlinear control based on robust right coprime factorization is presented for the Peltier actuated system in [4], where the plant and each controller are regarded as an operator, and signals are transferred from one space into another. Based on the design scheme, the realization of right coprime factorization controllers and design of robust 
tracking controller are discussed in [5]. Further, a control scheme is considered when the thermal system exist input constraints, where the designed controller is required to satisfy some robust right coprime factorization conditions [6]. Also, control and fault detection by using operator based nonlinear design scheme are investigated in [6-8]. Moreover, in $[9,10]$, operator based robust nonlinear control is designed for a multi-input multioutput thermal process with perturbation. However, the heat radiation will affect evidently the temperature of the Peltier actuated system when the temperature is high and temperature difference between the system and environment is large. A new nonlinear model with heat radiation needs to be built to design operator based robust right coprime factorization controllers. Also, in order to cancel the effect of the heat radiation, a compensation operator based on predictive model is investigated, where the model is considered by using radial basis kernel function based SVM method. SVM is a type of machine learning algorithm which is based on statistical learning theory and arises in recent years [11]. Comparing with SVM method and other machine learning methods, SVM can effectively improve generalization abilities of the algorithm, while not having a local minimum solution but a unique global optimal solution, and there are some characteristics, such as small sample learning ability and automatic identification of the model structure and the parameters [11-15]. It makes SVM very suitable for the modeling of the system with unknown structure and parameters according to the input and output data. Therefore, operator based robust nonlinear control with a compensation operator is put forward for the Peltier actuated system considering heat radiation in this paper.

The outline of this paper is given as follows. Section 2 begins with the introduction of the Peltier system, and modeling on the Peltier system and SVM based modeling on the heat radiation are also shown in this part. Design of operator based robust nonlinear controllers and compensation operator are proposed for the Peltier system in Section 3. Simulation results are given to illustrate the effectiveness of the modeling and the proposed scheme in Section 4. The conclusion is drawn in Section 5.

\section{Modeling}

2.1. Peltier Refrigeration System. The diagram of Peltier refrigeration system is shown in Figure 1, which mainly includes Advantech PCL812PG board, PCI1760U board, aluminium plate, Peltier actuator, temperature sensor, and PC. LM35 is used to measure the temperature of the aluminium plate, and its output voltage range is $0 \sim 1 \mathrm{~V}$. Peltier actuator is a kind of refrigeration device, which can produce cooling effect through changing duty cycle of PWM wave signal. PCL812PG board and PCI1760U board are inserted in PC, which are connected to the signal processing board by terminal boards. PCL812PG board with $16 \mathrm{~A} / \mathrm{D}$ channels and $4 \mathrm{D} / \mathrm{A}$ channels is selected to get temperature from sensor, whose available voltage range is $0 \sim 5 \mathrm{~V}$ with 12 -bit precision. PCI1760U board has 10 output channels with PWM wave, whose duty cycle is limited between $0 \%$ and $100 \%$. Signal processing board is

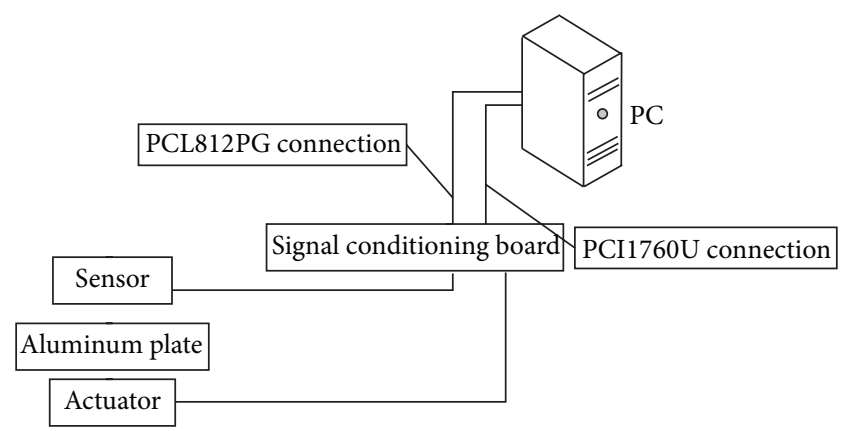

FIgURE 1: The diagram of Peltier refrigeration system.

designed to amplify voltage signal of the temperature sensor from $0 \sim 1 \mathrm{~V}$ to $0 \sim 5 \mathrm{~V}$ and convert the control signal $(5 \sim 12 \mathrm{~V})$ into PWM wave with different duty cycle. Control algorithm is realized and control signal is produced by PC.

In the refrigeration system, Peltier actuator is a semiconductor refrigeration device with composition of $N$-type semiconductor and $P$-type semiconductor. The heat flux and direction are controlled by the input current flow. Four main thermoelectric effects govern the thermodynamics of a Peltier actuator: the Seebeck effect, the Peltier effect, the Thomson effect, and the Joule effect. Due to the Peltier effect, heat will be absorbed at one end and pumped out through the other end when an external voltage is applied to a $N$-type/ $P$-type thermal couple. Thus, effectiveness of refrigeration can be realized by the Peltier actuator.

2.2. Modeling on the Peltier System. In order to set up the model of the Peltier system, some laws related to thermal exchange are utilized as follows [1].

(1) Fourier's law concerning thermal conduction is

$$
q\left[\mathrm{~W} / \mathrm{m}^{2}\right]=-\lambda[\mathrm{W} / \mathrm{mK}]\left(\frac{d \theta}{d n}\right)[\mathrm{K} / \mathrm{m}],
$$

where $q$ is heat flux, $\lambda$ is thermal conductivity, and $d \theta / d n$ is temperature gradient.

(2) Newton's cooling law is

$$
q\left[\mathrm{~W} / \mathrm{m}^{2}\right]=\alpha[\mathrm{W} / \mathrm{mK}](\Delta T)[\mathrm{K}],
$$

where $\alpha$ is thermal conductivity (flowing air: 10 600 [W/ $\mathrm{mK}]$ ) and $\Delta T$ is temperature difference.

(3) Stefan-Boltzmann law is

$$
\Phi[\mathrm{W}]=\varepsilon \sigma\left[\mathrm{W} / \mathrm{m}^{2} \mathrm{~K}^{4}\right] A_{S}\left[\mathrm{~m}^{2}\right] T^{4}\left[\mathrm{~K}^{4}\right],
$$

where $\Phi$ is radiation energy, $\varepsilon$ is emissivity $(\varepsilon<1), \sigma$ is radiation coefficient, $A_{S}$ is area, and $T$ is temperature.

(4) Thermal conduction law is

$$
d Q[J]=c[\mathrm{~J} / \mathrm{kgK}] m[\mathrm{~kg}] d \theta[\mathrm{K}],
$$

where $Q$ is Calorie heat, $c$ is specific heat, and $m$ is mass of the aluminum plate.

(5) Electrothermal amount on Peltier effect is

$$
\mathrm{Q}[\mathrm{J}]=S_{\mathrm{C}}[\mathrm{J} / \mathrm{KA}] T[\mathrm{~K}] I[\mathrm{~A}],
$$

where $S_{C}$ is Seebeck coefficient and $I$ is current. 


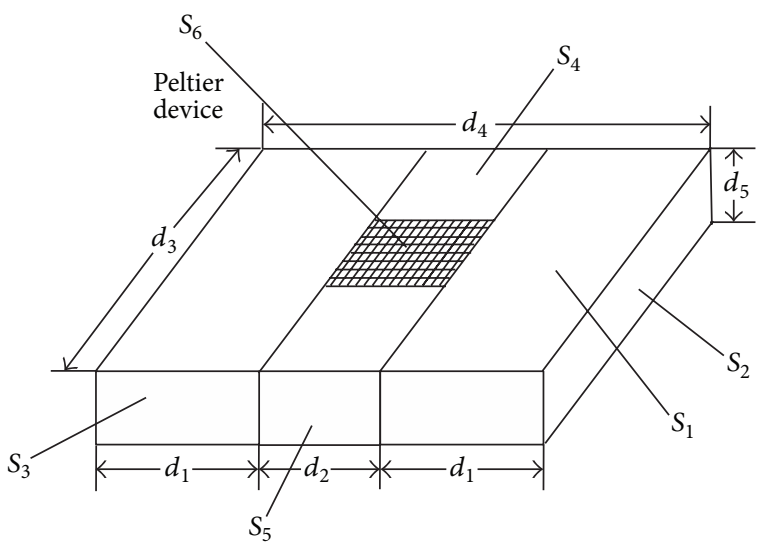

FIgURE 2: The aluminum plate thermal process with a Peltier device.

(6) Joule exothermic heat on current is

$$
Q[\mathrm{~J}]=R_{P}[\Omega] I^{2}\left[\mathrm{~A}^{2}\right],
$$

where $R_{P}$ is Peltier's resistance.

The aluminum plate thermal process with a Peltier device is shown in Figure 2.

A differential equation based on the above laws is obtained for the Peltier system:

$$
\begin{aligned}
c m & \frac{d\left(T_{0}-T_{x}\right)}{d t} \\
= & u_{d}-\alpha\left(T_{0}-T_{x}\right)\left(2 S_{4}+2 S_{5}-S_{6}\right)-2 \lambda\left(T_{0}-T_{x}\right) \frac{S_{2}}{d_{1}} \\
& -\varepsilon \sigma\left(4 S_{1}+2 S_{2}+4 S_{3}+2 S_{4}+2 S_{5}-S_{6}\right)\left(T_{0}^{4}-T_{x}^{4}\right),
\end{aligned}
$$

where $T_{0}$ is the environmental temperature, $T_{x}$ is the temperature, and $u_{d}$ denotes the heat absorbed by the Peltier device. Some parameters of the Peltier system are given in Table 1.

In the model, $\alpha\left(T_{0}-T_{x}\right)\left(2 S_{4}+S_{5}-S_{6}\right)$ denotes the energy produced by thermal convection between the middle aluminum plate and the air. $2 \lambda\left(T_{x}-T_{0}\right) S_{2} / d_{1}$ is the conducted energy from the other two parts to the middle part. And $\varepsilon \sigma\left(4 S_{1}+2 S_{2}+4 S_{3}+2 S_{4}+2 S_{5}-S_{6}\right)\left(T_{0}^{4}-T_{x}^{4}\right)$ is the energy produced by the thermal radiation. The endothermic amount of the Peltier device $u_{d}$ is shown in

$$
u_{d}=S_{C} T_{1} I-K\left(T_{h}-T_{1}\right)-\frac{R_{P} I^{2}}{2},
$$

where $T_{1}$ is the temperature of endothermic side, $T_{h}$ is the temperature of radiation side, and $I$ is the current flow. $S_{C} T_{1} I$ denotes the amount of heat that moves from the endothermic side to the radiation side by Peltier effect, $K\left(T_{h}-T_{1}\right)$ shows the movement heat caused by temperature difference between the two sides of the device, and $R_{P} I^{2} / 2$ is Joule heat which is generated by the input current through the device.

2.3. SVM Based Modeling on the Heat Radiation Part. In the above model, the heat radiation part contains the fourth power of the temperature, and it is not easy to be solved
TABle 1: Parameters of the Peltier system.

\begin{tabular}{lc}
\hline$I$ & Current: $0 \sim 2.2[\mathrm{~A}]$ \\
$u_{d}$ & Endothermic amount $[\mathrm{W}]$ \\
$c$ & $900[\mathrm{~J} / \mathrm{kg} / \mathrm{K}]$ \\
$\alpha$ & $15\left[\omega / \mathrm{m}^{2} / \mathrm{K}\right]$ \\
$\lambda$ & $238[\omega / \mathrm{m} / \mathrm{K}]$ \\
$\sigma$ & $5.6697 \times 10^{-8}\left[\mathrm{~W} / \mathrm{m}^{2} / \mathrm{K}^{4}\right]$ \\
$K$ & $0.63[\mathrm{~W} / \mathrm{K}]$ \\
$m$ & $d_{3} \times d_{4} \times d_{5} \times d[\mathrm{~kg}]$ \\
$d_{1}$ & $0.086[\mathrm{~m}]$ \\
$d_{2}$ & $0.068[\mathrm{~m}]$ \\
$d_{3}$ & $0.1[\mathrm{~m}]$ \\
$d_{4}$ & $0.24[\mathrm{~m}]$ \\
$d_{5}$ & $0.005[\mathrm{~m}]$ \\
$d$ & $2700\left[\mathrm{~kg} / \mathrm{m}^{3}\right]$ \\
$S_{C}$ & $0.053[\mathrm{~V} / \mathrm{K}]$ \\
$S_{1}$ & $d_{1} \times d_{3}\left[\mathrm{~m}^{2}\right]$ \\
$S_{2}$ & $d_{3} \times d_{5}\left[\mathrm{~m}^{2}\right]$ \\
$S_{3}$ & $d_{1} \times d_{5}\left[\mathrm{~m}^{2}\right]$ \\
$S_{4}$ & $d_{2} \times d_{3}\left[\mathrm{~m}^{2}\right]$ \\
$S_{5}$ & $d_{2} \times d_{5}\left[\mathrm{~m}^{2}\right]$ \\
$S_{6}$ & $9 \times 10^{-4}\left[\mathrm{~m}^{2}\right]$ \\
$R_{P}$ & $5.5[\Omega]$ \\
\hline
\end{tabular}

directly. Also, the heat radiation affects evidently the temperature of the Peltier actuated system when the temperature is high and temperature difference is large between the system and environment. In order to cancel the effect of the heat radiation, a compensation operator on the heat radiation is designed, which is based on a predictive model of the heat radiation. In this paper, the predictive model is set up by using SVM method and used to estimate the heat radiation. That is, SVM based predictive model on the heat radiation needs to be built.

SVM is firstly put forward by Vapnik, like multilayer perception network and radial basis function network, which can be used for pattern classification and nonlinear regression [11]. The basic idea of SVM is to create a classification hyperplane as decision surface which makes the isolation edge between positive examples and counter examples maximized. Its regression can construct an identification model of the system according to input and output data under the condition of some unknown parameters. SVM theory is based on Vapnik-Chervonenkis' statistical learning theory, where a structural risk minimization principle is utilized to get a unique global optimal solution. It can avoid local minimum problem and there are some good promotion characteristics, such as learning ability of small sample and automatic identification of structure and parameters of the model [12-15]. It is suitable for building the nonlinear predictive model of the heat radiation.

The detailed modeling on the heat radiation is shown as follows. Firstly, we define

$$
A=\frac{\left[\alpha\left(2 S_{4}+2 S_{5}-S_{6}\right)+2 \lambda S_{2} / d_{1}\right]}{(\mathrm{cm})},
$$




$$
\begin{aligned}
& M_{\text {svm }} \\
& =\frac{\varepsilon \sigma\left(4 S_{1}+2 S_{2}+4 S_{3}+2 S_{4}+2 S_{5}-S_{6}\right)\left(T_{0}^{4}-T_{x}^{4}\right)}{(\mathrm{cm})} .
\end{aligned}
$$

Then (7) is transformed as the following form:

$$
\frac{d\left(T_{0}-T_{x}\right)}{d t}=\frac{u_{d}}{c m}-A\left(T_{0}-T_{x}\right)-M_{\mathrm{svm}}
$$

It shows that the term $M_{\text {svm }}$ denotes the heat radiation part, which contains the fourth power of the temperature. Therefore, SVM method is considered to set up the model of $M_{\text {svm }}$ in this paper, and it is used to estimate the heat radiation real time. SVM concerning the estimation of a real-valued function can be described as follows:

$$
f(\tilde{x})=w^{T} \cdot \phi(\tilde{x})+b, \quad w \in R^{d}, b \in R .
$$

The model is based on a finite number set of independent and distributed data $\left(\tilde{x}_{i}, \tilde{y}_{i}\right)$, where $x_{i}$ and $y_{i}$ denote input and output, respectively, $w$ is weight vector, and $b$ is offset. In this paper, we make $u_{d}$ and $T_{x}$ as the input and $M_{\text {svm }}$ as the output when we build the SVM modeling, and $w$ and $b$ are identification model parameters after training. In Vapnik's $\varepsilon$-insensitive support vector regression, the aim is to find a function $f(\tilde{x})$ which allows error of $y_{i}$ to be no more than $\varepsilon$ and makes $y_{i}$ flat for all the training data. Considering more interferential errors, nonnegative slack variables $\xi$ and $\xi^{*}$ are introduced. Then, the optimization problem on the modeling can be described as the following form:

$$
\begin{array}{ll}
\min _{w, b} & \frac{1}{2} w^{T} \cdot w+C \sum_{i=1}^{l}\left(\xi_{i}+\xi_{i}^{*}\right) \\
\text { s.t. } & \left\{\begin{array}{l}
\tilde{y}_{i}-\left(w^{T} \cdot \phi\left(\tilde{x}_{i}\right)+b\right) \leq \varepsilon+\xi_{i} \\
\left(w^{T} \cdot \phi\left(\tilde{x}_{i}\right)+b\right)-\tilde{y}_{i} \leq \varepsilon+\xi_{i}^{*} \\
\xi_{i}, \xi_{i}^{*} \geq 0, i=1, \ldots, l,
\end{array}\right.
\end{array}
$$

where $C$ is a positive constant and used to control the punishment to the samples beyond the error $\varepsilon$. Generalizing to kernel function based regression estimation by introducing Lagrange multipliers, we can arrive at the following optimization problem [15]:

$$
\begin{gathered}
\max _{\alpha, \alpha^{*}} W\left(\alpha, \alpha^{*}\right)=-\frac{1}{2} \sum_{i=1}^{l} \sum_{j=1}^{l} Q_{i j}\left(\alpha_{i}-\alpha_{i}^{*}\right)\left(\alpha_{i}-\alpha_{j}^{*}\right) \\
+\sum_{i=1}^{l} \tilde{y}_{i}\left(\alpha_{i}-\alpha_{i}^{*}\right)-\sum_{i=1}^{l} \varepsilon\left(\alpha_{i}+\alpha_{i}^{*}\right) \\
\text { s.t. }\left\{\begin{array}{l}
\alpha_{i}, \alpha_{i}^{*} \in[0, C], i=1, \ldots, l \\
\sum_{i=1}^{l}\left(\alpha_{i}-\alpha_{i}^{*}\right)=0 \\
K\left(\tilde{x}_{i}, \tilde{x}_{j}\right)=\phi\left(\tilde{x}_{i}\right) \cdot \phi\left(\tilde{x}_{j}\right)=Q_{i j} .
\end{array}\right.
\end{gathered}
$$

Ultimately, the regression estimation takes the form

$$
\begin{aligned}
f(\tilde{x}) & =\sum_{i=1}^{l}\left(\alpha_{i}-\alpha_{i}^{*}\right)\left(\tilde{x}_{i} \cdot \tilde{x}\right)+b \\
& =\sum_{i=1}^{l}\left(\alpha_{i}-\alpha_{i}^{*}\right) K\left(\tilde{x}_{i} \cdot \tilde{x}\right)+b
\end{aligned}
$$

where $K\left(\tilde{x}_{i}, \tilde{x}_{j}\right)=\phi\left(\tilde{x}_{i}\right) \cdot \phi\left(\tilde{x}_{j}\right)$ is kernel function that returns the dot product in feature space by using a nonlinear mapping $\phi$ when two vectors are given in input space. That is, linear regression in a high dimensional space corresponding to nonlinear regression in the low dimensional input space can be realized by mapping the input vectors into a feature space. That is, nonlinear regression problem is resolved by linear regression form.

There are many kernel functions used in SVM, such as linear kernel function, polynomial function, radial basis function, and sigmoid function. Linear kernel can be looked as a special kind of form of RBF kernel function. The characteristic of sigmoid function is similar to RBF. In the radial basis function case, the support vector algorithm automatically determines centers, weights, and threshold such that radial basis kernel function is used in this paper, which is shown as follows:

$$
K\left(\tilde{x}_{i}, \tilde{x}\right)=\exp \left(-\frac{\left\|\tilde{x}_{i}-\tilde{x}\right\|^{2}}{2 \sigma^{2}}\right) .
$$

Therefore, under the above case, some parameters of support vector algorithm can be automatically determined, such as weight vector $w$, offset $b$, slack variables $\xi_{i}, \xi_{i}^{*}$, and Lagrange multipliers $\alpha_{i}$ and $\alpha_{i}^{*}$. Then, SVM model based estimation accuracy just depends on a setting of parameters $C$, $\varepsilon$, and $\sigma . C$ is penalizing constant, which determines the tradeoff between the model complexity and fatness. $\varepsilon$ is error-accuracy parameter, and it controls the width of the $\varepsilon$-insensitive zone for fitting the training data. $\sigma$ is kernel width parameter, which is appropriately selected to reflect the input range of the training data. By selecting the appropriate parameters $C, \varepsilon$, and $\sigma$, the predictive model of the heat radiation part $M_{\text {svm }}$ can be obtained and the heat radiation can be estimated in real time by the built predictive model. Then, operator based robust nonlinear control with compensation operator is presented for the Peltier system by the following section, where the compensation operator is proposed by using the SVM based predictive model.

\section{Robust Nonlinear Control with Compensation Operator}

3.1. Operator Based Robust Right Coprime Factorization. Robust right coprime factorization of a nonlinear system is to ensure that the system can be right coprime factorized and the system remains its right coprime factorization while it is affected by external perturbation. More specifically, we suppose that the nonlinear system $P$ has right factorization $P=N D^{-1}$, where $N$ and $D^{-1}$ are right factorization operators 


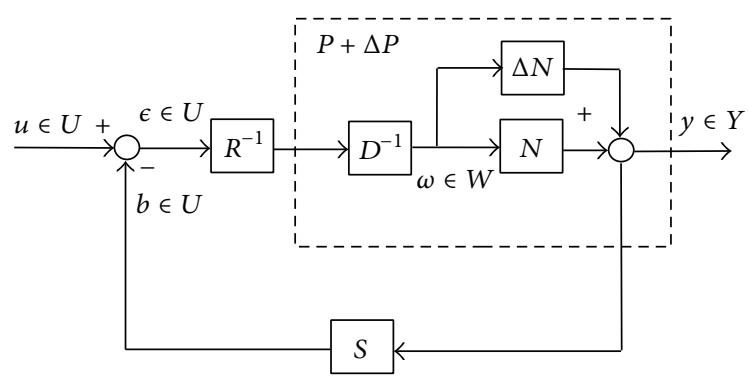

FIGURE 3: Robust right coprime factorization.

and the operation between $N$ and $D^{-1}$ means that the output of $D^{-1}$ is the input of $N$. Then, if there are two operators $S$ and $R$ satisfying Bezout identity $S N+R D=I$, we can say that right coprime factorization factors of the system exist and the factorization is right coprime. Assume that the system $P$ is disturbed by a perturbation $\Delta P$. If $P+\Delta P$ has a right coprime factorization and satisfies some conditions, it shows that the system $P$ has robust right coprime factorization shown in Figure 3.

The system $P$ without disturbance has the decomposition $P=N D^{-1}$. We define a disturbance operator as follows while $P$ is disturbed by $\triangle P$ :

$$
\Delta N=\Delta P D .
$$

So, for the stable operator $N+\Delta N, P+\Delta P$ can be factorized into

$$
P+\Delta P=(N+\Delta N) D^{-1} .
$$

Therefore, the system $P$ has the robust right coprime factorizations when the following conditions of robust stability are satisfied [3]:

$$
\begin{gathered}
S N+R D=L, \quad \text { where } L \text { is unimodular operator, } \\
\left\|(S(N+\Delta N)-S N) L^{-1}\right\|<1 .
\end{gathered}
$$

Based on the above robust right coprime factorization technology, a robust nonlinear control method with compensation operator is proposed for the Peltier actuated system.

\subsection{Robust Right Coprime Factorization with Compensation} Operator. For the Peltier system shown in (10), we define $y(t)=T_{0}-T_{x}$. The model can be reexpressed as

$$
\frac{d y}{d t}=\frac{u_{d}}{c m}-A y-M_{\mathrm{svm}},
$$

where the term $M_{\text {svm }}$ denotes the heat radiation part which can be estimated in real time by using the SVM based predictive model. In a word, the $M_{\mathrm{svm}}$ can be regarded as a predictive operator of the heat radiation part.

Considering the heat radiation as the perturbation, the Peltier actuated system can be expressed by (20):

$$
y=\frac{1}{c m}\left(e^{-A t}+\Delta\right) \int u_{d}(t) e^{A t} d t .
$$

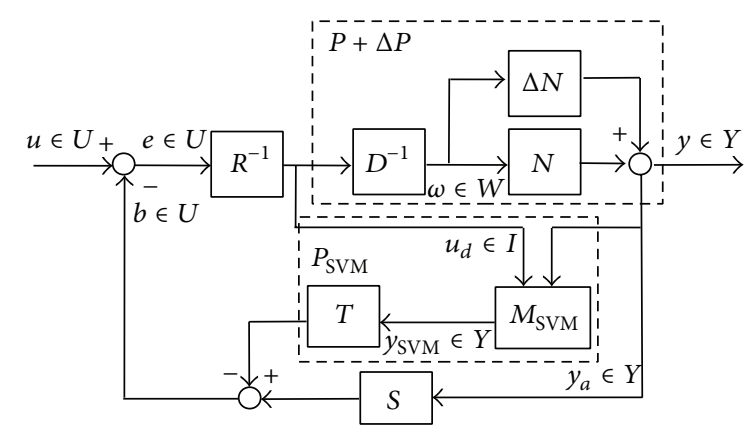

FIGURE 4: Robust nonlinear control scheme with compensation operator.

In order to cancel the effect of the heat radiation, a compensation operator $P_{\text {svm }}$ is designed according to the predictive operator $M_{\mathrm{svm}}$ and the transformer operator $T$. Robust right coprime factorization with the compensation operator is shown in Figure 4 for the Peltier system.

In Figure $4, U, Y$, and $W$ are the input space, output space, and quasistate space, respectively. The operator $M_{\mathrm{svm}}$ is designed by using the SVM based predictive model and $T$ is designed to transform the signal $y_{\text {svm }}$ from output space $Y$ to input space $U$.

Theorem 1. Assume that $\Delta \widetilde{N}=S^{-1} T M(D, N)$ is stable, where $M(D, N)$ denotes $M_{\text {svm }}(D(\omega), N(\omega))$. Let $S(N+\Delta N-\Delta \widetilde{N})-$ $S N$ be stable and invertible operator $(W \rightarrow U)$. The robust stability of the Peltier system considering heat radiation is ensured if the following equations and inequality are satisfied:

$$
\begin{gathered}
S N+R D=I, \\
S(N+\Delta N-\Delta \widetilde{N})+R D=\widetilde{L}, \\
\|(S(N+\Delta N-\Delta \widetilde{N})-S N)\|<1 .
\end{gathered}
$$

Proof. Under the assumption $\Delta \widetilde{N}=S^{-1} T M(D, N)$, the equivalent diagram of Figure 4 is obtained as shown in Figure 5. From (21) and (22), we have

$$
\widetilde{L}=I+S(N+\Delta N-\Delta \widetilde{N})-S N .
$$

Since $S(N+\Delta N-\Delta \widetilde{N})-S N$ is stable operator and $\|(S(N+$ $\Delta N-\Delta \widetilde{N})-S N) \|<1$, then $\widetilde{L}$ is invertible based on [3]. That is, $\widetilde{L}^{-1}=(I+S(N+\Delta N-\Delta \widetilde{N})-S N)^{-1}$. Since $S(N+\Delta N-\Delta \widetilde{N})-$ $S N$ is stable and invertible operator, and $I$ is identity operator, we have that $\widetilde{L}$ is also a unimodular operator. Therefore, the stability of operators $S, N, R, D, \Delta N$, and $\Delta \widetilde{N}$ can ensure that the outputs are bounded when the input signals are bounded in Figure 4.

So, the overall stability of the system is proved.

The nominal Peltier system is right divided into two parts $N$ and $D$ satisfying $P=N D^{-1}$ :

$$
\begin{gathered}
D^{-1}\left(u_{d}\right)=\frac{u_{d}(t)}{c m}, \\
N(\omega)(t)=e^{-A t} \int e^{A t} \omega(\tau) d \tau .
\end{gathered}
$$




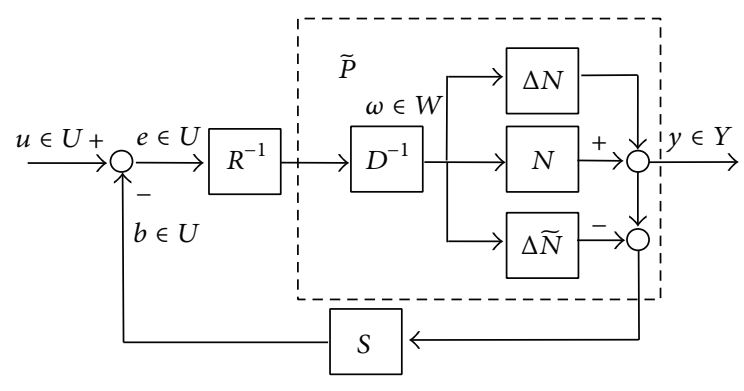

FIgURE 5: The equivalent diagram of Figure 4.

Considering the perturbation $\Delta P$ caused by the heat radiation, the corresponding perturbed operator $N+\Delta N$ can be obtained as

$$
(N+\Delta N)(\omega)(t)=\left(e^{-A t}+\Delta\right) \int e^{A t} \omega(\tau) d \tau
$$

which is bounded and stable. The uncertain term $\Delta$ is related to the heat radiation.

For the nonlinear system, nonlinear operator controllers $S$ and $R$ are designed to satisfy the Bezout identity (21) and inequality (23). It is worth to mention that the initial state should also be considered; that is, $S N\left(\omega_{0}\right)\left(t_{0}\right)+R D\left(\omega_{0}\right)\left(t_{0}\right)=$ $I\left(\omega_{0}\right)\left(t_{0}\right)$ should be satisfied. In this paper, we select the initial time $t_{0}=0$ and $\omega_{0}=\omega_{0}\left(t_{0}\right)$. By considering the structure of $D$, the controller $R$ is designed as

$$
R\left(u_{d}\right)=\frac{B u_{d}(t)}{c m},
$$

where $B$ is a constant. Then we can get

$$
R D(\omega)=R(D)=R(c m \omega(t))=B \omega(t)
$$

which shows that the expression of the $R D$ is simple. After that, it will be easy to get the expression of controller $S$. From Figure 4, we know that

$$
S N=S(N)=(1-B) \omega(t) .
$$

Based on $N(\omega)(t)=e^{-A t} \int e^{A t} \omega(\tau) d \tau$, we can get the following operator:

$$
S(y)=(1-B)\left(\frac{A y(t)+d y(t)}{d t}\right) .
$$

The predictive operator $M_{\text {svm }}$ is realized by using the SVM based predictive model introduced in Section 2. The transformer operator $T$ is

$$
T\left(y_{\mathrm{svm}}\right)=(1-B) c m y_{\mathrm{svm}}(t) .
$$

Furthermore, the tracking filter $M$ is designed to make the system output $y$ track the reference input $r$ shown in Figure 6. According to the Bezout identity (21), Figure 6 is equivalent to Figure 7. Using the result in [5], the tracking filter $M$ is designed as the following operator:

$$
M(r)(t)=(N+\Delta N)^{-1}(r)(t) .
$$

In the following section, the proposed control scheme is confirmed by simulation.

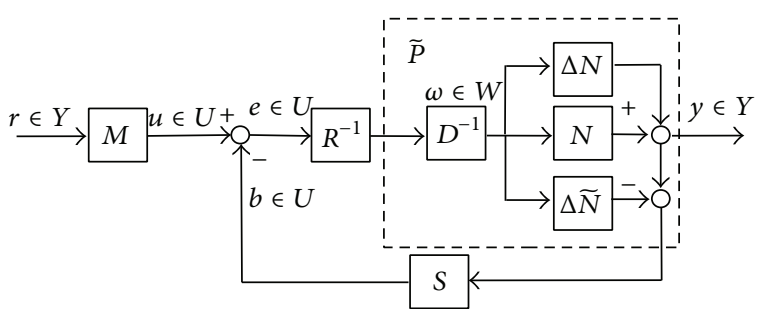

FIGURE 6: Robust tracking control scheme.

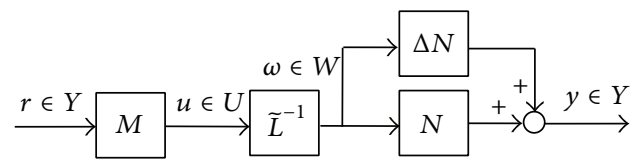

Figure 7: The equivalent diagram of Figure 6.

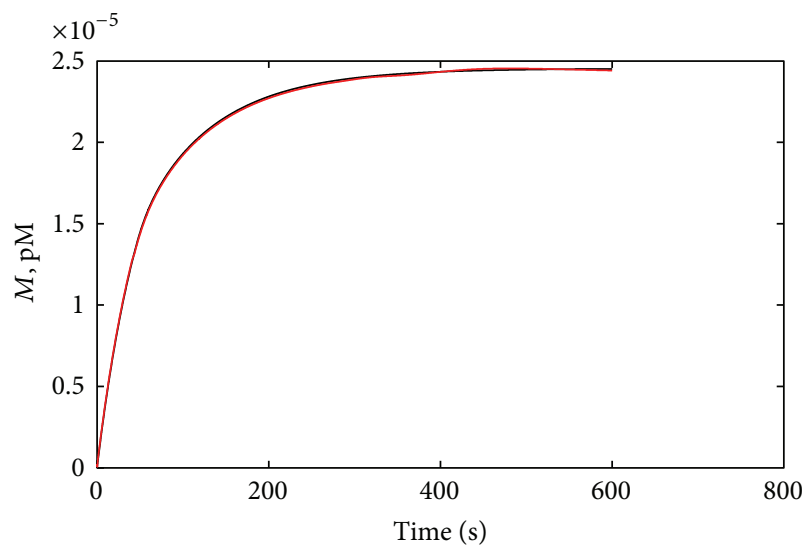

FIGURE 8: The prediction result of the heat radiation using SVM method.

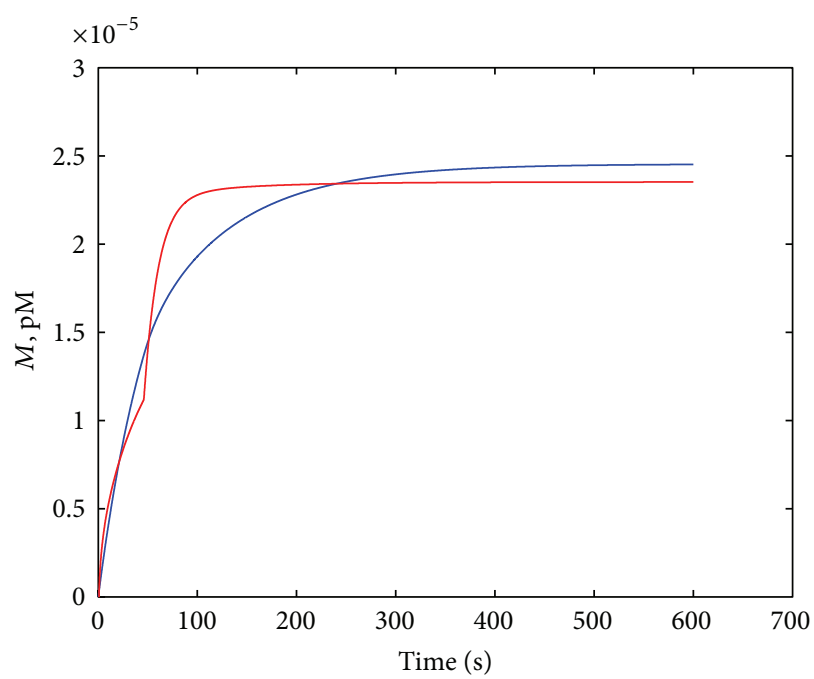

FIGURE 9: The prediction result of the heat radiation using least square method. 


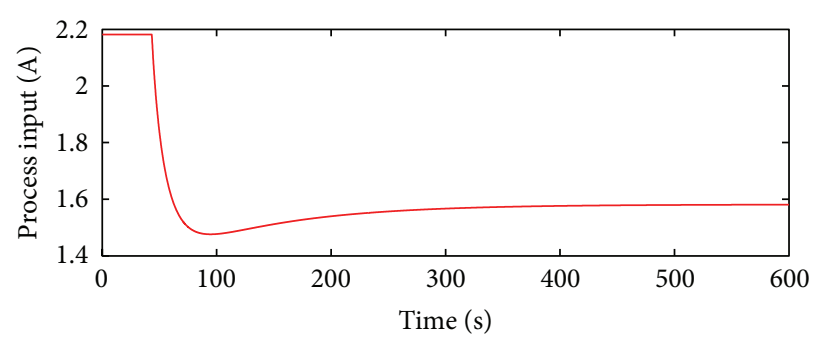

(a)

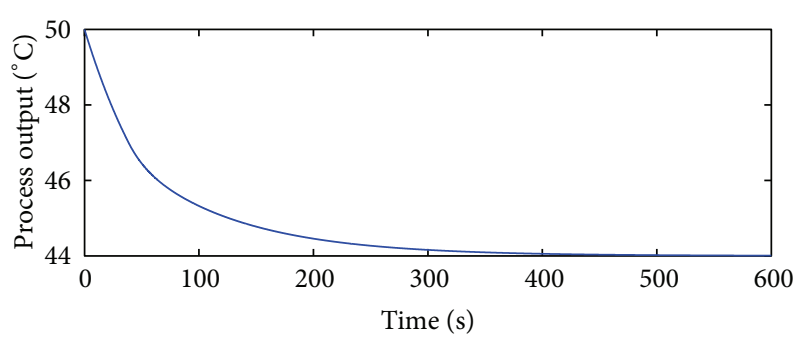

(b)

FIGURE 10: Simulation result without heat radiation.

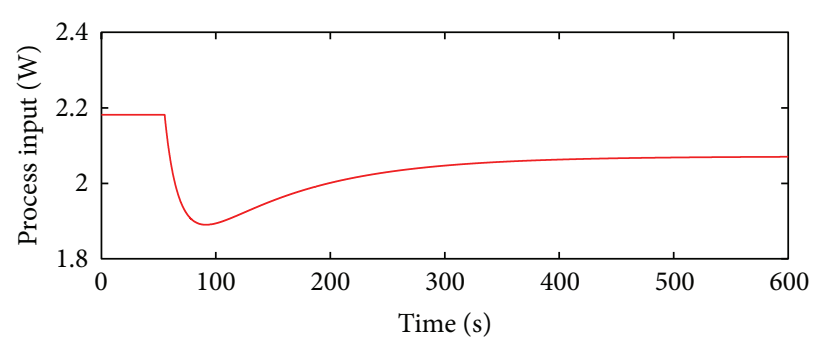

(a)

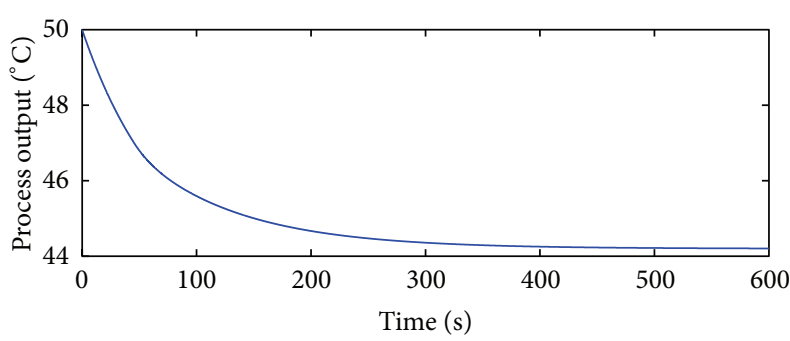

(b)

FIGURE 11: Simulation result with heat radiation.

\section{Simulation Results}

The system with Peltier device is a typical nonlinear control system considering the heat radiation. Because the heat radiation is related to the fourth power of temperature and is affected evidently when the temperature is high and temperature difference between the system and environment is large, a high initial temperature $\left(50^{\circ} \mathrm{C}\right)$ is settled to show the effectiveness of the control scheme. The input current is limited between $0.0[\mathrm{~A}]$ and 2.2[A]. The parameters used in the simulation are shown in Table 2 . In order to show the simulation results clearly, we change the unit of temperature " $\mathrm{K}$ " into " $\mathrm{C}$ " in the simulation.

4.1. Simulation on the Prediction Model. In this paper, the radial basis function is used to establish the predictive model $M_{\text {svm }}$ according to the SVM method, where some parameters of SVM are automatically determined, such as weight vector $w$, offset $b$, slack variables $\xi_{i}, \xi_{i}^{*}$, and Lagrange multipliers $\alpha_{i}$, $\alpha_{i}^{*}$. For the important design parameters $C, \varepsilon$, and $\sigma$, we set $2 \sigma^{2}$ as 0.1 , and $C$ and $\varepsilon$ are 100 and 0.1 , respectively.

According to the real data of the physical system, the predictive model of the heat radiation is obtained by using the SVM method. Figure 8 shows the prediction results of the heat radiation using the built predictive model. The black line is the real value of the heat radiation and the red one is the predicting result of the heat radiation using the SVM based predictive model. Its standard deviation and maximum deviation are $4.3815 \times 10^{-11}$ and $1.005 \times 10^{-7}$, respectively.

It is clear to see from Figure 8 that the two curves are almost coincident. Moreover, the heat radiation part is estimated by using the least square method based predictive model shown in Figure 9. Its standard deviation
TABLE 2: Simulation parameters.

\begin{tabular}{lc}
\hline Reference input (desired temperature) & $r=6\left[{ }^{\circ} \mathrm{C}\right]$ \\
Parameter & $B=0.99$ \\
Current & $I=0.0 \sim 2.2[\mathrm{~A}]$ \\
Initial temperature & $50\left[{ }^{\circ} \mathrm{C}\right]$ \\
Simulation time & $600[\mathrm{sec}]$ \\
Sampling time & $100[\mathrm{msec}]$ \\
\hline
\end{tabular}

and maximum deviation are $1.2905 \times 10^{-8}$ and $3.9561 \times$ $10^{-6}$, respectively. So it can be concluded that the SVM based predictive model of the heat radiation is more efficient and exact. The obtained model can be used to design the compensation operator.

4.2. Simulation on Robust Nonlinear Control with Compensation Operator. Firstly, in order to show effect of the heat radiation in the refrigeration system, the simulations are carried out for the system considering the heat radiation or not, which is simulated by the model. The parameters used in the two simulations are the same and the controllers without compensation operator are designed. Figure 10 shows the simulation result where the heat radiation has not been considered. And Figure 11 shows the simulation result where the heat radiation has been included.

From Figure 10, we can see that the output temperature of the system declined from $50^{\circ} \mathrm{C}$ to $44^{\circ} \mathrm{C}$ and finally falls in $44^{\circ} \mathrm{C}$. But in Figure 11, the output temperature of the system cannot decline to the $44^{\circ} \mathrm{C}$, and it just falls in $44.3^{\circ} \mathrm{C}$ at last. So it can be concluded that the heat radiation of the aluminum plate influences the output temperature of 


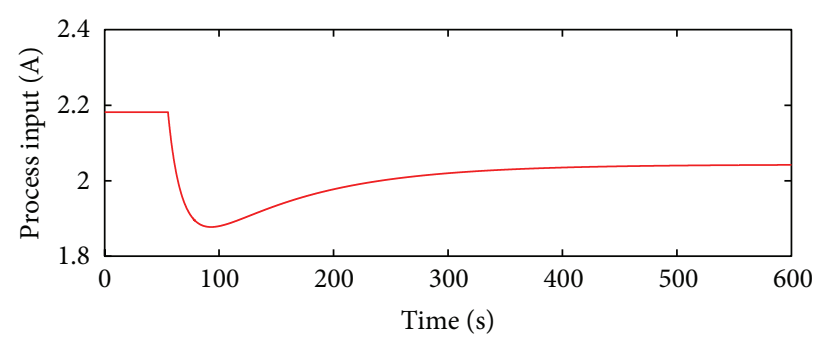

(a)

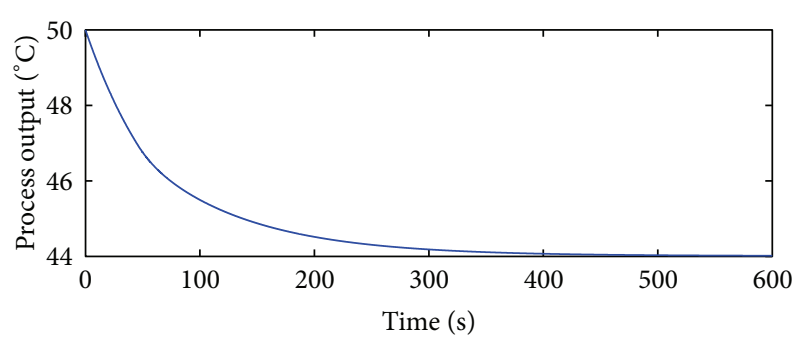

(b)

FIGURE 12: Simulation results using the proposed scheme.

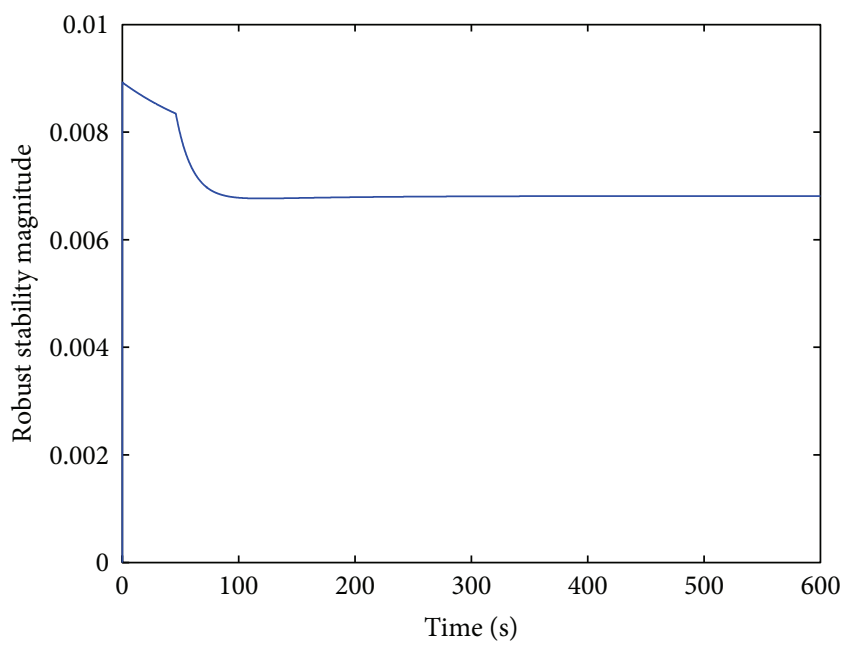

FIGURE 13: Result of robust stability analysis.

the system when the temperature is much higher than the environment temperature.

In order to cancel the effect of the heat radiation, robust right coprime factorization control with compensation operator is designed to make the system reduce to $44^{\circ} \mathrm{C}$. The simulation results are shown in Figure 12, which is similar to Figure 10. It shows that the temperature of the aluminum plate considering the heat radiation can be fallen in $44^{\circ} \mathrm{C}$ through using the proposed controllers. Comparing with Figures 10 and 12, it also can be concluded that the designed compensation operator based on the predictive model of the heat radiation and the proposed scheme are efficient.

Figure 13 shows the result of robust stability analysis considering the heat radiation. In Figure 13, the ordinate axis denotes robust stability magnitude based on a time sequence, which is calculated according to the robust stability condition (23). In Figure 13, the result shows that the robust stability magnitude is less than 1 . That is, the robust stability condition (23) is satisfied and the control system is robust stable.

\section{Conclusions}

For the Peltier actuated aluminum plate thermal system, it is certified that heat radiation influences evidently the temperature when the temperature is high and temperature difference is large between the system and environment. The model of the Peltier actuated system with heat radiation is built, and a predictive model on the heat radiation is obtained by using SVM method. Based on the predictive model, a compensation operator is designed to cancel the effect of the heat radiation. Furthermore, operator based robust nonlinear control with the compensation operator is presented to guarantee the robust stability and tracking performance for the Peltier actuated system. Finally, simulation results show the effectiveness of the proposed scheme.

\section{Conflict of Interests}

The authors declare that there is no conflict of interests regarding the publication of this paper.

\section{Acknowledgments}

This work is partially supported by National Natural Science Foundation of China (61074022 and 61304115) and Program for International Science Cooperation and Communication (2010DFA22770). Moreover, The authors declare that they have no financial and personal relationships with other people or organizations that can inappropriately influence their work, and there is no professional or other personal interests of any nature or kind in any product, service, and/or company that could be construed as influencing the position presented in, or the review of, the paper entitled.

\section{References}

[1] J. A. Chavez, J. A. Ortega, J. Salazar, A. Turo, and M. J. Garcia, "SPICE model of thermoelectric elements including thermal effects," in Proceedings of the 17th IEEE Instrumentation and Measurement Technology Conference (IMTC '00), vol. 2, pp. 1019-1023, Baltimore, Md, USA, May 2000.

[2] J. Jiang, G. V. Kaigala, H. J. Marquez, and C. J. Backhouse, "Nonlinear controller designs for thermal management in PCR amplification," IEEE Transactions on Control Systems Technology, vol. 20, no. 1, pp. 11-30, 2012.

[3] M. Deng, A. Inoue, and K. Ishikawa, "Operator-based nonlinear feedback control design using robust right coprime factorization," IEEE Transactions on Automatic Control, vol. 51, no. 4, pp. 645-648, 2006.

[4] M. Deng, S. Wen, and A. Inoue, "Operatorbased-robust nonlinear control for a Peltier actuated process," Measurement and Control, vol. 44, no. 4, pp. 116-120, 2011. 
[5] N. Bu and M. Deng, "System design for nonlinear plants using operator-based robust right coprime factorization and isomorphism," IEEE Transactions on Automatic Control, vol. 56, no. 4, pp. 952-957, 2011.

[6] M. Deng, A. Inoue, and K. Edahiro, "Fault detection in a thermal process control system with input constraints using a robust right coprime factorization approach," Proceedings of the Institution of Mechanical Engineers I: Journal of Systems and Control Engineering, vol. 221, no. 6, pp. 819-831, 2007.

[7] M. Deng, A. Inoue, and K. Edahiro, "Fault detection system design for actuator of a thermal process using operator based approach," Acta Automatica Sinica, vol. 36, no. 4, pp. 580-585, 2010.

[8] S. Wen and M. Deng, "Operator-based robust nonlinear control and fault detection for a Peltier actuated thermal process," Mathematical and Computer Modelling, vol. 57, no. 1-2, pp. 1629, 2013

[9] S. Bi and M. Deng, "Operator-based robust control design for nonlinear plants with perturbation," International Journal of Control, vol. 84, no. 4, pp. 815-821, 2011.

[10] M. Deng and S. Bi, "Operator-based robust nonlinear control system design for MIMO nonlinear plants with unknown coupling effects," International Journal of Control, vol. 83, no. 9, pp. 1939-1946, 2010.

[11] V. N. Vapnik, Statistical Learning Theory, John Wiley \& Sons, New York, NY, USA, 1998.

[12] H. Drucker, C. J. C. Burges, and L. Kaufman, "Support vector regression machines," in Advances in Neural Information Processing Systems, pp. 155-161, MIT Press, Cambridge, UK, 1997.

[13] J. K. A. Suykens, T. V. Gestel, and J. D. Brabanter, Least Squares Support Vector Machines, World Scientific Publishing, Singapore, 2002.

[14] S. Wen, M. Deng, and A. Inoue, "Operator-based robust nonlinear control for gantry crane system with soft measurement of swing angle," International Journal of Modelling, Identification and Control, vol. 16, no. 1, pp. 86-96, 2012.

[15] M. Deng, L. Jiang, and A. Inoue, "Mobile robot path planning SVM and lyapunov function compensation," Measurement and Control, vol. 42, no. 8, pp. 234-237, 2009. 


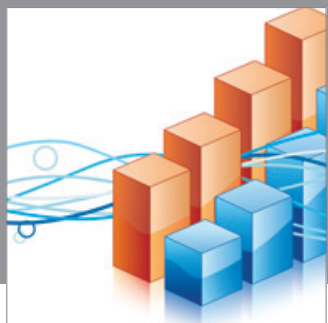

Advances in

Operations Research

mansans

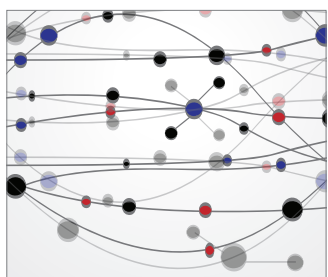

The Scientific World Journal
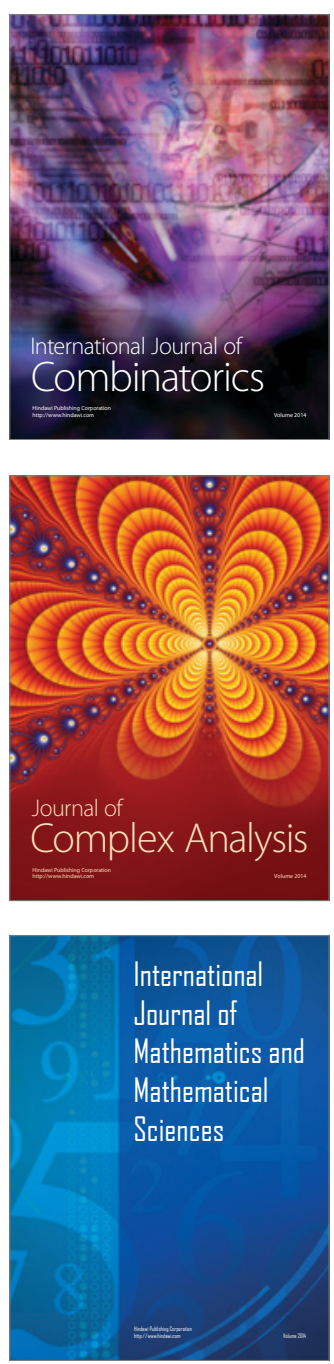
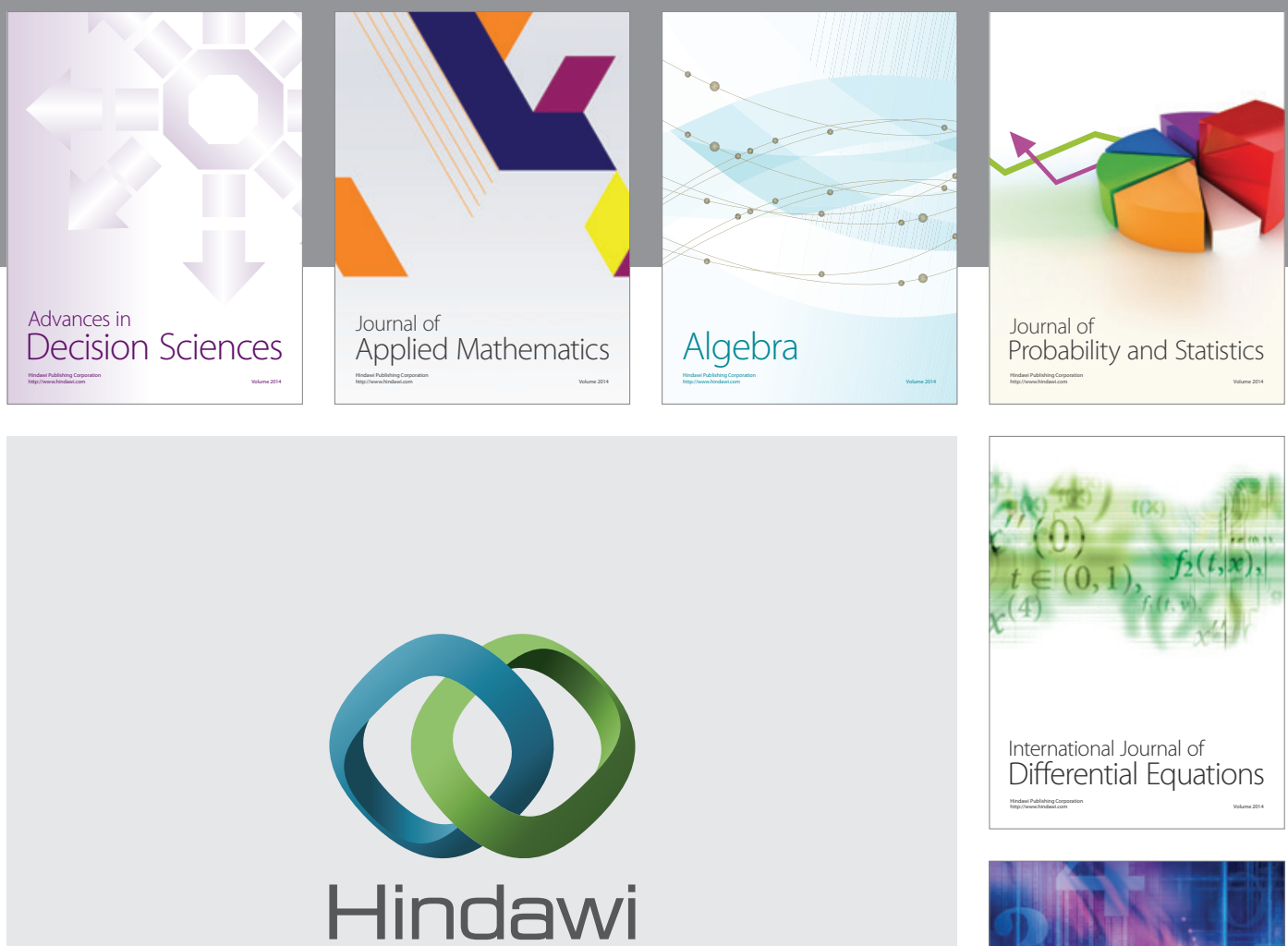

Submit your manuscripts at http://www.hindawi.com
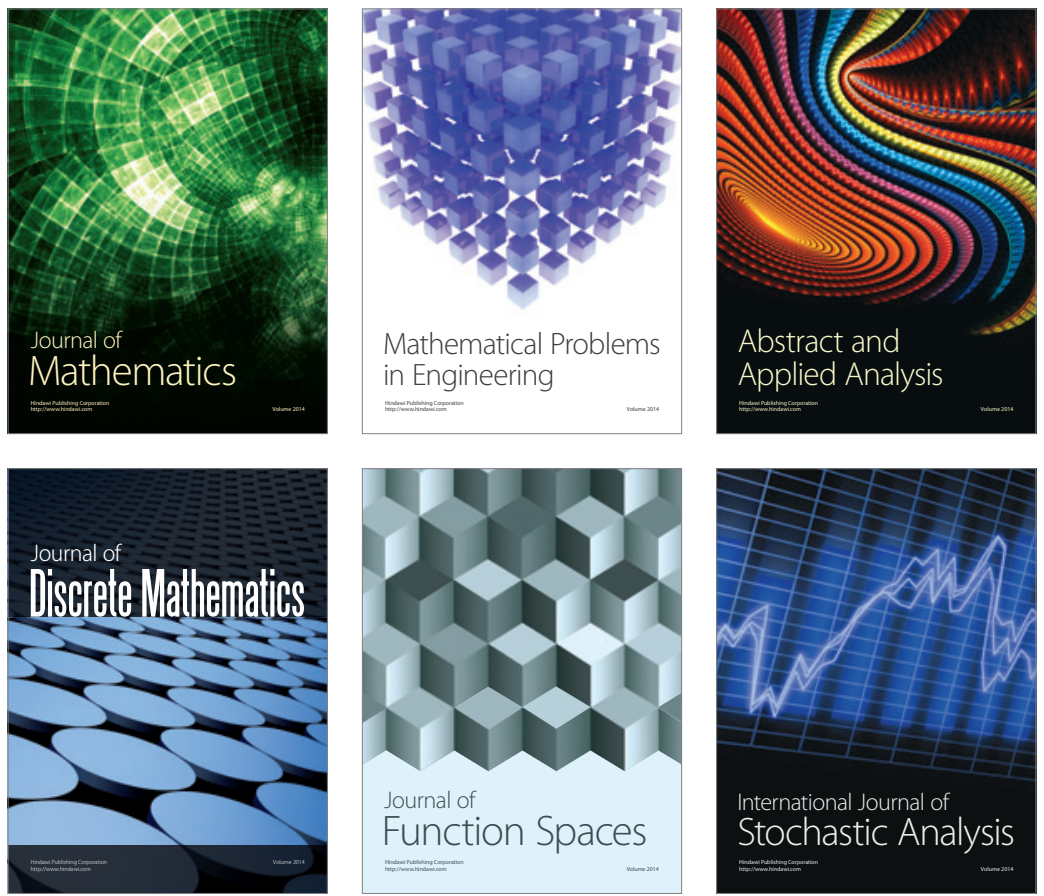

Journal of

Function Spaces

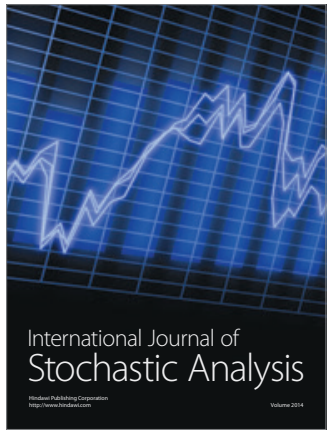

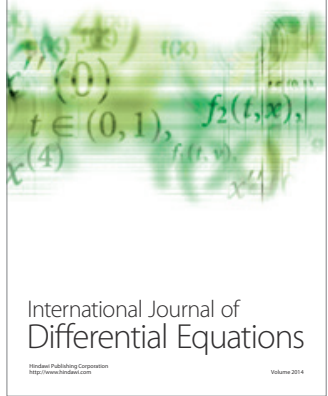
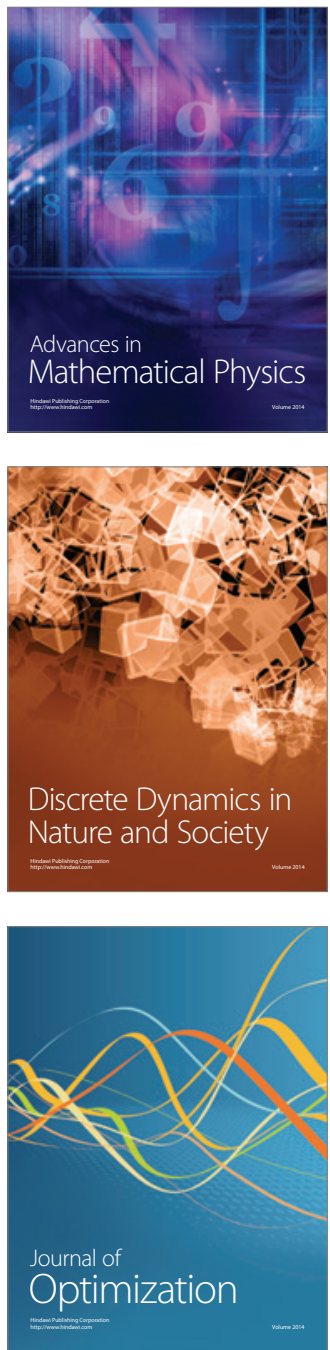